\title{
WELT-VORGEGEBENHEIT UND IHRE DEUTUNGEN: VON HUSSERL ZU EINER KOSMOLOGISCHEN WENDE UND ZURÜCK ${ }^{1}$
}

KAREL NOVOTNÝ

\section{Abstract \\ The pre-givenness of the world and its interpretation: From Husserl to a cosmological turn and back again}

The article follows in its first part some basic ideas of Edmund Husserl concerning the pre-givenness of the world. In the genetic analysis the world in the naturality of its self-evident being presents an enigma for Husserl indeed that he tried to reduce by elucidating the ways how it is (pre-)given in and through horizonal world-apperceptions. The second part of the article mentions some limits of this approach that Eugen Fink has sharply seen, already as an assistant of Husserl, and it outlines briefly a turn towards another approach to the world as developed later by Fink in his "kosmology". In the third part the article makes reference to a recent project of a phenomenological metaphysics by László Tengelyi who re-actualized a Husserlian approach to the world's pre-giveness as one of the originary facta (Urtatsachen) of the experience instead to resorb this and other originary facta of experience by a kosmological or other speculative turn into a unique movement of the world or being itself. Tengelyi is presented as an exemple of a renewal of phenomenology where in a return from the kosmology back to Husserl the world becomes "the proper issue of phenomenology" again.

Die Welt als „die eigentliche Sache der Phänomenologie“, um einen gegenwärtigen Autor, Klaus Held, zu zitieren, ${ }^{2}$ ist mit einem der wichtigsten, bis heute le-

1 Der vorliegende Aufsatz ist in Zusammenhang mit dem Grant-Projekt „Leben und Umwelt. Phänomenologische Bezüge zwischen der Subjektivität und der natürlichen Welt“ (GAP 15-10832S) entstanden.

2 Held Klaus, „Die Endlichkeit der Welt. Phänomenologie im Übergang von Husserl zu Heidegger“, in Niemeyer B. und Schütze D. (Hrsg.), Philosophie der Endlichkeit. Festschrift für Erich Christian Schröder zum 65. Geburtstag, Würzburg, Königshausen und Neumann, 1992, S. 130.

https://doi.org/10.14712/24646504.2019.5

(C) 2019 The Author. This is an open-access article distributed under the terms of the Creative Commons Attribution License (http://creativecommons.org/licenses/by/4.0). 
bendigen philosophischen Ansätze Edmund Husserls verbunden. Seine lebendige Gegenwärtigkeit ist auch hier, wie üblich, mit Revisionen, Umkehrungen, ja Häresien verbunden. So nahm die Welt-Problematik bei Martin Heidegger, Eugen Fink, Maurice Merleau-Ponty und anderen Philosophen, die sich eingehend kritisch mit Husserls transzendental-philosophischem Ansatz auseinandergesetzt haben, neue Wege, bisweilen bis hin zu einer Art kosmologischer Wende. Die produktive Auseinandersetzung mit solchen Herausforderungen, die bis heute anhalten - wie z. B. bei Renaud Barbaras -, führten aber durchaus auch zu Versuchen, das phänomenologische Welt-Denken Husserls zu erneuern und die Welt als phänomenologische Sache selbst zurückzugewinnen.

\section{Husserl}

Der Zugang zur Welt-Problematik wird bei Edmund Husserl mit einem zentralen Konzept - dem Begriff des Horizontes - verbunden. Im Spätwerk Husserls heißt es dazu: „Die Konstitution der vorgegebenen Welt systematisch auslegen das ist systematisch die Horizontstruktur derselben auslegen“"3.

Damit sind andere, ebenso zentrale Motive genannt. Erstens der Charakter der Vorgegebenheit, der dem phänomenologischen Weltbegriff, wie er anhand der Horizontstruktur der Erfahrung beschrieben wird, wesentlich eignet. Zweitens die Art und Weise, wie mit der Aufklärung des Sinnes dieser Welt in ihrer Vorgegebenheit umgegangen wird: Es geht um eine systematische Auslegung der Konstitution dieses Sinnes, was auf die transzendental-phänomenologische Methode hinweist, die jeden Sinn, auch den „vorgegebenen“ Sinn der Welt, die selbst kein Gegenstand ist, sondern ein alle Gegenstände Umfassendes bedeutet, auf seine Korrelation zu Sinnstiftungen und Sinnbildungen im Bewusstsein bezieht. ${ }^{4}$

3 Husserl Edmund, Die Lebenswelt. Auslegungen der vorgegebenen Welt und ihre Konstitution. Texte aus dem Nachlass (1916-1937), Dordrecht, Springer, HUA XXXIX, 2008, S. 125.

4 Vgl. den Aufsatz „Die Welt als philosophisches Problem“, der Ende der 1930er Jahre von Ludwig Landgrebe verfasst wurde. Zuerst 1940 in englischer Übersetzung erschienen (in Philosophy and Phenomenological Research I, 1940, S. 38-58), dann auf Deutsch in: Landgrebe Ludwig, Der Weg der Phänomenologie. Das Problem der ursprünglichen Erfahrung, Gütersloh, Gütersloher Verlagshaus, 1963, S. 41 ff. Er hat ihn in einer Zeit angefertigt, in der er neben der Arbeit an der Verfassung von Erfahrung und Urteil u. a. intensiv darum bemüht war, einen Band zum Thema „Welterfahrung und Horizontprobleme“ zur Publikation für Husserl in Prag vorzubereiten. Im September 1938 schrieb Jean Héring an Hedwig Conrad-Martius: „Von des Meisters Manuskripten ist der erste Band im Druck: Erfahrung und Urteil. In Aussicht: Bd II: Welterfahrung und Horizontprobleme. III. Zeitprobleme. IV. Anthropologie usw." Zitiert bei R. Sowa in der Einleitung zu HUA XXXIX, Op. cit., S. LXI, Anm. 1. 
In gebotener Kürze soll dieser Horizont-Begriff umrissen werden, den Husserl sachlich schon früh - nämlich im Zusammenhang mit den Analysen der Wahrnehmung und des Zeitbewusstseins - entdeckte und dann zum ersten Mal systematisch im ersten Buch seiner Ideen zu reinen Phänomenologie und phänomenologischen Philosophie (Ideen I) präsentierte. Diese Analysen ergaben u. a., dass kein Bewusstsein von etwas isoliert erlebt werden kann. Es impliziert immer schon Hinweise auf unterschiedliche Sinn-Zusammenhänge, sowohl in Bezug auf das aktuell bewusste Objekt selbst als dessen sogenannten inneren Horizont (seiner näheren Bestimmungen) als auch in Bezug auf die Situiertheit dieses Objektes im Verhältnis zu anderen Objekten als seinen äußeren Horizont (etwa im Raum). Horizonte sind dabei sowohl auf Seiten der Noesis (der Akte des Bewusstseins selbst) als auch auf Seiten der Noema, also der gemeinten Gegenständlichkeiten impliziert. Alle diese inneren und äußeren, noetischen und noematischen $\mathrm{Zu}$ sammenhänge und Verweisungen in ihren gegenseitigen Verbindungen und Verschachtelungen können ebenso als Horizonte gefasst und prinzipiell in der Analyse ausgewiesen werden.

Eine Bestimmung der Welt lässt sich bereits in Anknüpfung an diese Analysen phänomenologisch aufzeigen, dass nämlich die Welt als Horizont all jener Horizonte angegangen werden kann, die in Korrelation mit Bewusstseinsakten einzelner, allerding immer schon vergemeinschaftlichter Subjektivitäten stehen. Doch wird es sich dabei immer nur um eine wesentliche Bestimmung der Erfahrung handeln: Das aktuell Bewusste hebt sich stets vom Hintergrund der impliziten Sinnzusammenhänge ab, die, da sie immer mitgegeben sind, als Potenzialitäten des Bewusstseins des Aktuellen expliziert werden können. Man kann schon hier in einem gewissen Sinne von einer „Vorgegebenheit" der Horizonte sprechen, die die einzelnen Erfahrungen umfassen.

Im Zusammenhang mit dem Thema der Epoché und der transzendentalen Reduktion, die Husserl ebenfalls zuerst in den Ideen I der Öffentlichkeit vorlegte, wird eine wesentliche Bestimmung der Welt selbst entdeckt, nämlich die Funktion einer Generalthesis, durch welche die Welt immer schon durch die einzelnen Objektsetzungen mit- und vorausgesetzt wird. Um alle in der natürlichen Einstellung des Bewusstseins "naiv“ gehegten Meinungen über das zu erkennende Sein der Objekte insgesamt einzuklammern bzw. außer Geltung zu setzen, stößt die Epoché, die diese Einklammerung zu leisten hat, auf eine grundlegendere, unthematische und generelle Glaubensgewissheit eines Ganzen: die sogenannte Boden-Funktion der Welt. Sie wird vor allem im Rahmen der genetischen transzendentalen Phänomenologie später (seit Mitte der 1920er Jahre) weiter untersucht und gedeutet. 
Damit wird die Vorgegebenheit der Welt in ihrer Unmodalisierbarkeit sichtbar: Während jede Einzelerfahrung modalisiert werden kann, indem sich ihr Objekt etwa im Laufe der Erfahrung als Illusion enthüllt, ist die Welterfahrung als solche "nicht modalisierbar" . Darin bekundet sich die Nichtreduzierbarkeit der "Generalthesis" der Welt, ja eine Urfaktizität der Welthabe, und zwar deshalb, weil diese Funktion, so Landgrebe, selbst „kein bestimmter Akt“ ist, der „,irgendwo einmal ausdrücklich vollzogen worden wäre“, sondern „die Grundlage aller bestimmten Akte $[. .$.$] als eine Urtatsache, dass wir von vorneherein und ganz selbst-$ verständlich ,in eine Welt hineinleben'..." ${ }^{\prime}$.

Diese Bestimmung der Welt-Vorgegebenheit als Urtatsache weist darauf hin, dass sich auch in der genetischen Analyse diese Funktion der Welt-Thesis nicht aus den einzelnen Erfahrungen mit den Objekten (etwa als erworbene Habitualitäten) ableitet, ganz so, wie die Horizonte, die in der Tat die jeweils aktuellen Lebens-Umwelten konkret mit ausmachen: „Vielmehr alle Habitualitäten, die dem Menschen angeboren oder von ihm im Laufe seines Lebens erworben sind, sind Habitualitäten seiner als des Menschen, der schon auf dem Boden des Weltglaubens steht und sich in ihm als ein Seiendes unter Seiendem weiß"7.

Wenn es aber so ist, dass sich dieser Glaube von der Horizontbildung selbst unterscheidet, obwohl durch sie hindurch die Weltlichkeit jeder Erfahrung bewirkt wird - wo kommt der Weltglaube im Erleben dann her? Dieser Glaube als die Urtatsache der Weltbezogenheit ist ja auch ein Erlebnis, daher muss an ihn ebenfalls die Frage nach der Genesis oder dem Ursprung des Weltglaubens gestellt werden können.

In seinem letzten Werk, der Krisis der europäischen Wissenschaften, schreibt Husserl selbst: So

lebt der Phänomenologe in der Paradoxie, das Selbstverständliche als fraglich, als rätselhaft ansehen zu müssen und hinfort kein anderes wissenschaftliches Thema haben zu können als dieses: die universale Selbstverständlichkeit des Seins der Welt - für ihn das größte aller Rätsel - in eine Verständlichkeit zu verwandeln. ${ }^{8}$

Davon, dass es sich dabei um keine isolierte Formulierung handelt, zeugt etwa auch die folgende Stelle aus demselben Werk:

5 Husserl E., Die Lebenswelt. Auslegungen der vorgegebenen Welt und ihrer Konstitution. Texte aus dem Nachlass (1916-1937), HUA XXXIX, op. cit., S. 246.

6 Landgrebe L., Der Weg der Phänomenologie, op. cit., S. 44 f.

7 Ibid., S. 45.

8 Husserl E., Die Krisis der europäischen Wissenschaften und die transzendentale Phänomenologie, Den Haag, Martinus Nijhoff Verlag, HUA VI, 1976, S. 184. 
Für den Transzendentalphilosophen ist aber die gesamte reale Objektivität, die wissenschaftliche Objektivität aller wirklichen und möglichen Wissenschaften, aber auch die vorwissenschaftliche der Lebenswelt mit ihren ,Situationswahrheiten' und der Relativität ihrer seienden Objekte, nun zum Problem, zum Rätsel aller Rätsel geworden. Das Rätsel ist gerade die Selbstverständlichkeit, in der für uns beständig und vorwissenschaftlich ,Welt' ist, als Titel für eine Unendlichkeit von allen objektiven Wissenschaften unentbehrlichen Selbstverständlichkeiten. ${ }^{9}$

Eugen Fink spricht das Welt-Rätsel in seinem Aufsatz „Edmund Husserl in der gegenwärtigen Kritik“ aus den Kant-Studien aus dem Jahre 1933 auf folgende Weise an:

Wenn die erste und notwendig vorläufige Bestimmung des Wesens der natürlichen Einstellung als Weltgläubigkeit, als die universale strömende Weltapperzeption, sich auch innerhalb ihrer selbst, d. i. auf dem Boden dieses Glaubens selbst, vollzieht, so ist dabei das Entscheidende das Erwachen einer maßlosen Verwunderung über die Rätselhaftigkeit dieser Sachlage. Sie als selbstverständliche Tatsache hinnehmen, heißt Blindbleiben für das erste aller Rätsel, für das Sein der Welt selbst, als die aus der strömenden Weltapperzeption allererst Sinn und Seinsgeltung empfangende Welt und das mit allem je erdenklichen Inhalt, in dem sie jeweils für uns ist. ${ }^{10}$

Welt-Vorgegebenheit und ihre Deutung bei Husserl erfolgen in einem transzendental-philosophischen Rahmen, der vermutlich bei keinem Mysterium ${ }^{11}$

9 Ibid., S. 208. Jan Patočka bezeichnet in seinem Aufsatz „Zur Vorgeschichte der Wissenschaft von der Bewegung: Welt, Erde, Himmel und die Bewegung des menschlichen Lebens“ aus dem Jahre 1964 diesen grundlegenden Bezug auf das Weltganze als das „Mysterium der Selbstverständlichkeit“: „Und man kann nicht außer Betracht lassen, dass schon die Tatsache einer nicht-sinnlichen Anwesenheit des sinnlich Zugänglichen schwindelerregend ist; dass sie die Tür zu einem gewissen Mysterium der Selbstverständlichkeit öffnet, dessen wir uns nicht bewusst sind, da wir mit ihm gleichsam zusammengewachsen sind, uns in ihm bewegen, leben und sind. Das menschliche Leben ist also durch ein vorgängiges Ganzes charakterisiert." In Patočka Jan, Die Bewegung der menschlichen Existenz, hg. v. K. Nellen, J. Němec u. I. Srubar, Stuttgart, Klett-Cotta Verlag, $134 \mathrm{f}$.

10 Fink Eugen, „Edmund Husserl in der gegenwärtigen Kritik“, in Kant-Studien 38 (1933), S. 321-383. Wiederabgedruckt in: Fink Eugen, Studien zur Phänomenologie, Den Haag, Martinus Nijhoff Verlag 1966. Hier S. 115 f. Dabei bildet „die Epoché [...] das transzendentale Problem der Welt, das Grundproblem der Phänomenologie", ibid., S. 119.

11 Maurice Merleau-Ponty wird dann in seinem Aufsatz „Der Philosoph und sein Schatten“ von 1959 über „das Mysterium einer Weltthesis vor allen Thesen“ schreiben. Vgl. ders., Signes, Paris, Les Éditions Gallimard, 1960, S. 227. Dt. Übersetzung: Merleau-Ponty Maurice, Zeichen, Hamburg, Meiner, 2007, S. 239. Zitiert bei Etienne Bimbenet, der festhält: „Da ist wirklich ein Mysterium, worüber sich das Werk Merleau-Pontys wundert auf jeder Seite.“ Vgl. Bimbenet Etienne, „Merleau-Ponty: la parole du monde“, in Alter. Revue de phénoménologie, Nr. 6: Monde(s), Paris, Editions Alter, 2006, S. 13; Übers. des Zitats K. N. 
wirklich Halt macht, sondern eher in immer ursprünglichere Schichten der Erfahrungsgenesis zurückfragt, um eine Aufklärung des Ursprungs der Welthabe durch genetische Aufklärung der Weltapperzeption zu gewinnen, so wie sie sich aufgrund der Affektionen in den Prozessen der Sinnbildung und Sinnstiftung einstimmig artikuliert. Diese Aufklärungen gehen, wie schon gesagt, von der Beschreibung der natürlichen Welthabe aus. ${ }^{12}$

Das zeigt die transzendental-genetische Deutung der Vorgegebenheit der Welt bei Husserl an, die man als seine Antwort auf die Frage nach dem Welt-Ursprung ${ }^{13}$ auffassen kann: Die Welt ist „als Gebilde“ der transzendentalen Subjektivität (vor-) konstituiert. ${ }^{14}$ Und diese Subjektivität ist daher auch so eng mit der Welt verbunden, dass erstere ohne letztere gar keinen Inhalt und die letztere ohne die erstere keinen Bestand hat. Die Welt ist Korrelat einer universalen Weltapperzeption, die im Leben der transzendentalen Subjektivität ihre Quelle hat. Über diese Korrelation geht die Phänomenologie Husserls nicht hinaus, sie ist ihr Absolutes. Mit Fink formuliert:

Die Erkenntnisbewegung, die in der Reduktion geschieht, ist zwar ein transzendierender Übergang (von der Welt zur ,transzendentalen Subjektivität'), aber prinzipiell innerhalb der Einheit des Absoluten, als welches wir im Durchgang durch die phänomenologische Philosophie das ,konstitutive Werden' der Welt aus den Ursprüngen des ,transzendentalen' Lebens erkennen. So wie die Welt nur ist, was sie ist, aus dem ,Ursprung her, so ist dieser selbst auch nur, was er ist, auf die Welt hin. ${ }^{15}$

12 Husserl formuliert so in einem seiner späten Forschungsmanuskripte: „Die Welt als Universum der einstimmig fortzuführenden Erfahrung ist für jeden normalen reifen Menschen, kann man sagen, wirklich konstituiert, eben als ausgebildete Vermöglichkeit, immer weiter erfahren zu können - das ist der ,Weltbegriff' des natürlichen menschlichen Lebens; der alltägliche Sinn ,Welt ' geschöpft aus der im Verwirklichen dieses Prozesses des ,Immer-wieder usw.' erwachsenden beweglichen ,Weltanschauung' oder erfahrungsmäßigen Anschauung von Welt." Husserl E., Späte Texte über die Zeitkonstitution (1929-1934). Die C-Manuskripte, hrsg. von Lohmar, D., Dordrecht, Springer, HUA Materialien VIII, 2006, S. 186, Anm. 2.

13 Noch einmal Eugen Fink: „Die Grundfrage der Phänomenologie, zu der sie von vielen, an traditionelle Probleme anknüpfenden Einsätzen her unterwegs ist und in der sich ihr radikaler Gegensatz zum Kritizismus offenbart, läßt sich formulieren als die Frage nach dem Ursprung der Welt." (Ibid., S. 101)

14 Die Absicht der transzendental-genetischen Frage nach dem Ursprung der Welt besteht nämlich, wie es Husserl programmatisch in der Krisis der europäischen Wissenschaften formuliert, darin, „verstehen [zu] lernen, dass die ständig in uns im strömenden Wandel der Gegebenheitsweisen seiende Welt ein universaler geistiger Erwerb ist, als das geworden und zugleich fortwerdend als Einheit einer geistigen Gestalt, als ein Sinngebilde - als Gebilde einer universalen letztfungierenden Subjektivität" (Husserl E., Die Krisis der europäischen Wissenschaften und die transzendentale Phänomenologie, op. cit, S. 115).

15 Fink E., Studien zur Phänomenologie, op. cit., S. 106. 
Dieses „auf die Welt hin“ beschreibt Husserl, ähnlich wie generell bezüglich des intentionalen „auf ein Objekt Gerichtet-seins“ eines jeden Bewusstseins, mit dem Begriff der Apperzeption: „Die Welt ist für uns da als vorgegebene, sofern schon vor der thematischen Blickrichtung auf die oder jene Objekte oder gar auf die Welt als Universum durch unser Leben beständig eine Weltapperzeption hindurchgeht" ${ }^{16}$. Erst dank dieser ,in der Passivität einig in einer fließenden und doch immerfort sich vereinheitlichenden Gesamtapperzeption "17 erscheinen immerfort Dinge, gleichgültig, „ob wir gerade auf sie achten oder nicht“ ${ }^{18}$. Durch diese „Gesamtapperzeption" ist uns die Welt horizontmäßig vorgegeben, und sowie wir die Reflexion ins Spiel bringen, werden wir durch Reflexion als Erfahrung dessen inne werden, dass das vorgegebene Weltliche „immerfort sein subjektives Milieu hat und ohne das uns nie bewusst werden kann" 19 .

Soweit zu der Art und Weise, wie bei Husserl die Vorgegebenheit der Welt - in der Reichhaltigkeit seiner Analysen und trotz anderen Deutungsmöglichkeiten, die sie anzubieten mögen - grundsätzlich gedeutet wird.

\section{Kosmologische Wende}

In der Einleitung von Erfahrung und Urteil lesen wir dementsprechend: „Die horizonthaft bewusste Welt hat in ihrer ständigen Seinsgeltung den subjektiven Charakter der Vertrautheit im allgemeinen ..."20. Aus einer kritischen Perspektive gesehen, steckt in einer solchen Beschreibung eine Tendenz, die Husserl vom frühen Fink als Vorurteil der Vorgegebenheit der Welt, und später als eine Art „metaphysische Klausur" zum Vorwurf gemacht werden wird. Die Tendenz nämlich, die Weltapperzeption so zu deuten, als könne sie vorschreiben, dass „das Ding [...] jedes Reale überhaupt als Erfahrbares sein allgemeines ,Apriori', eine Vorbekanntheit" hat und haben muss, indem sie sich im Vorhinein bis zu einer „Totalitätstypik“ erstreckt, und zwar so, dass diese Totalität „,zum ganzen Welthorizont in seiner Unendlichkeit“ gehöre, der alles Einzelne von vornherein umspielt und mitbestimmt. Die wesentliche Vertrautheit der Welt würde es so im Zuge der erwähnten Totalisierungs-Tendenz nahelegen, dass jede „Unbekanntheit [...] jederzeit zugleich Modus

\footnotetext{
6 Husserl E., Die Lebenswelt. Auslegungen der vorgegebenen Welt und ihrer Konstitution. Texte aus dem Nachlass (1916-1937), HUA XXXIX, op. cit., S. 42.

Idem.

Idem.

19 Ibid., S. 43.

20 Husserl E., Erfahrung und Urteil. Untersuchungen zur Genealogie der Logik, redigiert und herausgegeben von Ludwig Landgrebe, Claassen \& Goverts, Hamburg 1948, S. 33.
} 
der Bekanntheit ist“ (ebd., 34), der sich im Zuge der Objekt-Konstitution bildet und auf das Universum übertragen als eine Folie einer Vor-Bestimmtheit verbreitet. ${ }^{21}$

Das scheint für kritische Stimmen in der phänomenologischen Bewegung die Offenheit der Welt und vor allem des Erscheinens selbst zu unterbinden, was einerseits zu einem immer deutlicheren Abstand oder gar einer Abwendung von diesem apriorischen Welt-Rahmen führt: Für die „Linie“von Emmanuel Levinas über Michel Henry oder Jean-Luc Marion, und ebenfalls für Marc Richir, ist die Welt kein Grund des Erscheinens. Das Erscheinen ist hier nicht an die „in der Passivität einig in einer fließenden und doch immerfort sich vereinheitlichenden Gesamtapperzeption" gebunden, von der wir oben bei Husserl berichtet haben. Aber dieser Abkehr gingen andererseits bestimmte kosmologischen Wenden voraus, für welche das Erscheinen seinen Grund sehr wohl in der Welt hat. Beiden „Linien“ ist freilich gemeinsam, dass sie das Erscheinen vor seiner Auflösung in eine universale Weltapperzeption und letztlich in das bloße Korrelat des intentionalen Bewusstseins bewahren wollen. Ein diesbezüglicher Weg scheint darin zu liegen, die Welt in ihrer ursprünglicheren Vorgegebenheit zu retten oder sie gar als den übergeordneten Rahmen zu etablieren, der alle phänomenalen Korrelationen, das Erscheinen als solches, allererst ermöglicht.

Diesen Impuls, die Welt anders zu denken, ja, eine kosmologische Wende über die Phänomenologie hinaus anzusetzen, verfolgt - im Anschluss an Heideggers Kritik der Phänomenologie - Eugen Fink. Als enger Mitarbeiter Husserls hat Fink den Meister in seinen späten Gedankengängen und Projekten begleitet, sich dabei aber in seiner eigenen Philosophie auf andere Wege begeben. Eine zentrale Rolle spielte dabei zunehmend gerade der Anspruch, den Welt-Bezug des Menschen nicht erst vom intentionalen Bewusstsein und seinen Horizonten aus, sondern aus jenem Rahmen denkend anzusetzen, der ein solches Bewusstsein und seine Horizonte immer schon umgreift. So entstand der Anspruch, die Dimensionen, die in der Phänomenologie Husserls, aber auch bei Heidegger, insofern verloren gehen, als bei ihnen dieser Rahmen in Sinn-Zusammenhängen besteht und sich darauf beschränkt, neu einzuholen.

Im Zusammenhang mit unserem Aufriss der phänomenologischen Welt-Problematik, in dem wir die Welt-Vorgegebenheit und ihre Deutungen aufeinander beziehen, weist Klaus Held in seinem Aufsatz „Die Endlichkeit der Welt“ auf eine Einsicht hin, die einen wichtigen Schritt auf dem Weg hin zu einer phänomenologischen Kosmologie markiert. Sie charakterisiert den entscheidenden Schritt Hei-

21 Fink E., Welt und Endlichkeit, Würzburg, Königshausen \& Neumann, 1990, S. 148: „Für eine intentionale Interpretation, die ansetzt beim Ich-Ding-Bezug“, ist die „Gegend“, aus der die Gegenstände insgesamt begegnen, „etwas Abkünftiges, ist die immer weiter hinausgeschobene Näh““. 
deggers in Sein und Zeit über Husserl hinaus und stellt eine Antwort auf die oben erwähnte Schwierigkeit dar, die Welthabe transzendental-phänomenologisch aufzuklären. Sie lautet: Die Welt ist ursprünglich vorintentional vorgegeben aufgrund der Bereitschaft, „so oder anders affiziert zu werden“. Sie bestimmt das „Wie des in der Korrelation [...] [Bewusstsein - erscheinender Gegenstand] geschehenden Erscheinens im Ganzen"22.

Es ist daher wichtig, dass diese Bereitschaft nicht in der Verfügbarkeit des intentionalen "Ich kann“ des Bewusstseins steht, da sie dieses „Ich kann“ einerseits in seiner Freiheit einschränkt, ja vom Unverfügbaren abhängig macht - daher die Rede von der Endlichkeit der ursprünglichen, vorintentionalen Welt-Vorgegebenheit. Andererseits aber werden dieses „Ich kann“ und sein immer weiter ins Endlose der Welt Hineinschreiten-Können eben auch dadurch erst ermöglicht, dass ohne eine solche Bereitschaft, affiziert zu werden, das intentionale „Ich kann“ gar nicht geweckt werden kann. Daher schreibt Held: „So ist sie [Bereitschaft, affiziert zu werden] nichts anderes als die Weise, wie sich jeweils die verhältnislose Weltoffenheit selbst ereignet“23. Und er charakterisiert diese Bereitschaft mit Heidegger als „Befindlichkeit“, als „ursprüngliche stimmungshafte Weltoffenheit“. Diese ist wesentlich mit einem Entzug und der Endlichkeit der Welt verbunden, denn: „In der Stimmung überkommt uns der Entzug, der die Welt endlich sein lässt. Im Staunen fühlen wir, dass wir der Weltoffenheit nicht Herr sind [...]“, da diese sich im „Abgrund des Nichts [...] entziehen könnte" 24 .

Doch auch in dieser Darstellung der Endlichkeit der Welt bei Heidegger, die ihre Unendlichkeit im Sinne Husserls - die ins Endlose gehende Verschränkung der Horizonte - ermöglichen soll, bleibt die Welt ein Horizont, ein Sinn-Zusammenhang, auch wenn die Vorgegebenheit der Welt als eine vorintentionale keine Apperzeption ist, die bei Husserl letztlich das exklusive Muster der Gegebenheit bleibt. Es ist Eugen Fink, der anders noch als Heidegger die Welt in ihrem Entzug angeht und auslotet.

Nach seiner Assistenzzeit bei Husserl, also in späteren, eigenständigen Ausarbeitungen und Anläufen zu Kosmologie von einer solchen phänomenologischen Perspektive, wählt Fink nur mehr einen negativen Ausgang, wie etwa in der Vorlesung über Welt und Endlichkeit aus dem Jahre 1949: „Welt ist das paradoxe in-

22 Helds Absicht in diesem Aufsatz, in dem übrigens auch die eingangs zitierte These über die Welt als „die eigentliche Sache der Phänomenologie“ formuliert wird, besteht darin, Folgendes zu begründen: „Die im Sinne Heideggers verstandene Endlichkeit der Welt ermöglicht ihre Unendlichkeit im Sinne Husserls.“ (Held K., „Die Endlichkeit der Welt. Phänomenologie im Übergang von Husserl zu Heidegger", art. cit., S. 131)

23 Ibid., S. 141.

24 Ibid., S. 143. 
tentionale Phänomen eines aller Erfahrung vorausspringenden und sie im ganzen umstellenden Leerhorizontes. Welt gibt es für Husserl nicht an sich, so wenig wie für Kant. Sagt Kant, sie ist bloß ,Idee, so Husserl: sie ist bloß ,Horizont “" ${ }^{25}$. Nun gehe es darum, Folgendes einzusehen: „In ihm [im Horizont-Phänomen] meldet sich wohl die Welthaftigkeit der Welt, aber es kann und darf nicht mit der Welt gleichgesetzt werden; es gründet in der Offenheit der Welt, aber ist nicht die eigentlich welthafte Weise des Offenseins" ${ }^{\text {"26. }}$.

Durch ein Konzept des „Rückscheins“ löst sich Fink, der jedoch bereits in seiner Dissertation eine nicht horizonthafte Mitpräsenz des Bild-Trägers bei der Bildwahrnehmung mit dem Terminus des „Durchscheinens“ bezeichnete, „vom Modell der

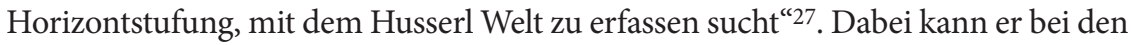
Horizonten ansetzen, aber diese sozusagen in umgekehrter Richtung lesen, als es bei Husserl geschieht: Die Erfahrungshorizonte sind so für Fink „nicht mehr als die in der Immanenz einer Subjektivität sich konstituierenden Außenbezüge ihrer selbst, sondern als in der Immanenz eines Lebensumkreises erfolgenden Ankündigungen von solchem“ zu lesen, „was diesen Umkreis übersteigt“ 28.

Was durch diese Umkehrung erreicht werden soll, ist eben eine andere Deutung der Vorgegebenheit der Welt als bei Husserl, der sie als den „einstimmige[n] Totalhorizont aller Erfahrung bestimmt", und diese andere Deutung der Vorgegebenheit kann anhand des „Rückscheins“ als ein Hereinstehen der Welt in das subjektive System der Einstimmigkeit der Horizonte gefasst werden:

Denn ein Horizontsystem ist nicht nur an einen (subjektiven) Träger geknüpft, sondern korreliert mit einer bestimmten Richtung, indem die Offenheit eines solchen Systems darin besteht, dass ihr Träger hinaussteht. Bei der Umkehrung des Systems, im Hereinstehen von Welt, findet sich der Träger in solches eingestellt, das aufgrund des geänderten Richtungssinns nicht eigentlich mehr als ein Ineinander von Horizonten bezeichnet werden kann..$^{29}$

Der Sinn von Horizont selbst wird überstiegen, weil es sich als ungenügend erweist, „den Sinn von Welt an den Horizontbezug zu binden“30.

Fink E., Welt und Endlichkeit, op. cit., S. 149.

Ibid., S. 29.

27 Nielsen Cathrin und Sepp Hans Rainer, „Welt bei Fink“, in Nielsen C. und Sepp H. R. (Hrsg.), Welt denken. Annäherungen an die Kosmologie Eugen Finks, Freiburg/München, Verlag Karl Alber, 2011, S. 10.

Idem.

Idem. 
Daher die wiederholten Versuche, aus der horizonthaften Weltapperzeption des intentionalen Bewusstseins weg zu einem anderem Denken vorzustoßen, wie in der bereits zitierten Vorlesung Welt und Endlichkeit aus dem Jahre 1949:

\begin{abstract}
Aber was noch viel entscheidender ist, auch nicht der bloße Abstoß vom Binnenweltlichen, das Hinausstehen über alles Seiende auf das umfangende Ganze zu, ist die rechte Bahn. Wo Welt, wo der Weltraum und die Weltzeit sozusagen nur angedacht werden, in der Negation des Binnenweltlichen, gemeint werden als das Umfangende, das vom Umfangenen wesensverschieden ist, dort kann, wenn es hochkommt, nur die Unerreichbarkeit der Welt erfahren werden als unerreichbar für das metaphysische Denken, das vom Seienden ausgeht - und sich vielleicht noch davon abstößt. [...] Die Selbstverdeckung von Raum und Zeit, ihr Sichzeigen am Binnenräumlichen und Binnenzeitlichen, ist gerade eine fundamentale Weise, wie sich die Welt dem Zugriff des metaphysischen Denkens entzieht. ${ }^{31}$
\end{abstract}

Auch dem Ansatz Heideggers bei dem stimmungsmäßigen Entzug der Welt stellt sich Fink daher kritisch entgegen, auch wenn er Heideggers Absage an die Metaphysik bis zu einer gewissen Grenze folgt. ${ }^{32}$

\title{
III. Zurück zur Welt als der Sache selbst der husserlschen Phänomenologie
}

Wir haben oben darauf hingewiesen, dass nach Klaus Held der Unendlichkeit des konkreten Welthorizontes des Erscheinens ein stimmungshafter Horizont der Endlichkeit - die Befindlichkeit - zugrunde gelegt werden kann, die allererst Offenheit für das Erscheinen ermöglicht. László Tengelyi verteidigte demgegenüber in sei-

31 Fink E., Welt und Endlichkeit, op. cit., 201 f. Im Blick auf seinen frühen „Absprung vom Transzendentalismus“ notierte er einmal: „Mein Weg war die Weltzeit als umgreifendes überobjektives und übersubjektives Urgeschehen.“ (Dagegen: „Husserl Ausweg aus diesen Problemen war der verstärkter Präsentialismus der ,lebendigen Gegenwart' (1930)“ (Fink E., „Fünf lose Blätter zur Zeitproblematik", in Bruzina Ronald (Hrsg.) Phänomenologische Werkstatt. Band 2: Bernauer Zeitmanuskripte Cartesianische Meditationen und System der phänomenologischen Philosophie, Freiburg/München, Verlag Karl Alber, Eugen Fink Gesamtausgabe, Band 3/2, 2008, S. 440).

32 Die Auseinandersetzung Finks mit Heideggers spätem Denken zu Erde und Physis aus seinem Vortrag Vom Ursprung des Kunstwerks aus dem Jahre 1935, der für Fink wie für viele andere kritische Weltdenker in der phänomenologisch orientierten Philosophie wegweisend wurde, können wir hier nicht mehr vorfolgen. Es sei jedoch auf die ausgezeichneten Aufsätze von Cathrin Nielsen und Françoise Dastur hingewiesen: Nielsen Cathrin, „Kategorien der Physis. Heidegger und Fink“ in Nielsen C. und Sepp H. R., Welt denken, op. cit., S. 154-183; Dastur Françoise, „Le concept du monde chez Heidegger après Etre et temps“ in Alter. Revue de phénoménologie, Nr. 6: Monde(s), Paris, Editions Alter, 2006, S. 119 ff. 
nem letzten Buch Welt und Unendlichkeit, das 2014 posthum publiziert wurde, ${ }^{33}$ die offene Unendlichkeit der Welt. Tengelyi deutet darin die Welt-Vorgegebenheit von einem metaphysischen Weltentwurf aus, der als eine Erweiterung der Phänomenologie konzipiert wird, die einerseits auf ihrem methodischen Transzendentalismus, ${ }^{34}$ andererseits auf der Anerkennung weiterer, unableitbarer Urtatsachen basiert:

Zum nicht-traditionellen Charakter dieser Metaphysik gehört, dass sie nicht nach ersten Gründen und Ursachen des Seienden als Seienden forscht. Vielmehr stützt sie sich von vornherein auf gewisse Urtatsachen. Nach Husserls Einsicht kann die phänomenologische Metaphysik - im Gegensatz zur transzendentalen Phänomenologie - keineswegs als eine apriorische Wissenschaft aufgefasst werden. Im Gegenteil, sie wird als eine Wissenschaft des Faktischen bestimmt“ ... wo „die ,Notwendigkeit eines Faktums' einen ,Kern des Urzufälligen " keineswegs" ausschließt. ${ }^{35}$

Die Metaphysik zufälliger Faktizität findet Tengelyi also bereits bei Husserl, sofern dieser die Urtatsachen kennt, die jeder eidetischen Variation und damit jeder eidetischen transzendentalen Phänomenologie zugrunde liegen. Tengelyi identifiziert zunächst vier derartige Urtatsachen in Husserls Schriften, fügt dann allerdings noch zwei weitere hinzu. Diese vier Urtatsachen sind das jeweilige Ich, die Welthabe dieses Ichs, das intentionale Ineinandersein von Ich und Anderen sowie das Faktum einer Geschichtsteleologie. ${ }^{36}$ Dazu treten an einer prominenten Stelle das Ereignis des Erscheinens selbst ${ }^{37}$ und - eher unauffällig oder unterschwellig - auch die als Urtatsache angenommene Leiblichkeit:

Die Urtatsachen, die Husserl nunmehr im Auge hat, verweisen auf Faktizitätstrukturen, die sich mit dem Cogito verbinden und ihm einen unverlierbaren Hinweis auf Welthabe, Leiblichkeit, Intersubjektivität und Geschichtlichkeit einprägen. In ihnen kommt das zum Ausdruck, was man mit Eugen Fink als eine, immanente Selbstüberschreitung der Egologie` bei Husserl bezeichnen könnte. ${ }^{38}$

Entscheidend ist dabei, dass diese Urtatsachen in ihrer Faktizität und Vielheit bestehen bleiben, ohne dass sie selbst noch einmal aus ersten Ursachen abgeleitet werden könnten. Es ist in ihnen keinerlei onto-theologische Grundstruktur aus-

\footnotetext{
3 Tengelyi László, Welt und Unendlichkeit. Zum Problem phänomenologischer Metaphysik, Freiburg/ München, Verlag Karl Alber, 2014.

34 Ibid., S. 200.

35 Ibid., S. 14.

36 Ibid., S. 184.

37 Ibid., S. 190.

38 Ibid., S. 186.
} 
zumachen, und doch handelt es sich nicht einfach um empirische Tatsachen unter anderen. ${ }^{39}$

Was die Urtatsache der Welthabe selbst und ihre Unendlichkeit betrifft, geht es Tengelyi um die Einführung eines „diakritische[n] Unterschied[es]“ der „grundlegende[n] Differenz zwischen Totalität und Unendlichkeit" ${ }^{40}$. In Bezug auf unsere Darstellung der Problematik der Deutung der Welt-Vorgegebenheit, von der wir ausgegangen sind - die bei Husserl, z. B. in Erfahrung und Urteil, Gefahr zu laufen scheint, die Welt restlos in das Korrelat des intentionalen, a priori vorbestimmenden Bewusstseins eingehen zu lassen und damit ihre Offenheit zu unterbinden und die Alterität oder den Entzug abzuschwächen -, bietet sich bei Tengelyi folgende Konstellation an: Das „Unendliche der Welt ist ein diesseitiges Unendliches“, es ist „nicht etwa das Weltall als geschlossenes Ganze gemeint", sondern es „handelt sich dabei vielmehr um die Offenheit der Welt für das Unendliche“, und zwar so, dass der „Welt [...] eine für das Unendliche offene Totalität" zukommt. Zwischen dieser Totalität und dem Unendlichen gibt es daher keinen Gegensatz, sondern „eben nur ein[en] Unterschied“41. Dieser Unterschied, der auf die sogenannte „diakritische Methode“ verweist, ist jedoch entscheidend, von ihm hängt die Chance ab, der erwähnten Gefahr, die Welt auf das Korrelat des Bewusstseins zu reduzieren, zu entkommen.

Phänomenologisch ist diese Methode bereits bei Merleau-Ponty, der Tengelyi inspiriert hat, vorgezeichnet, und zwar „in dem Sinne, dass [Merleau-Ponty] [...] gegenüber einem reinen Strukturdenken in der Dritte-Person-Perspektive Distanz wahrt und nach meine[r] Einschaltung in ein universelles diakritisches System “42 fragt. Die Nähe zu Husserls phänomenologischer Idee der „Dingstruktur im unendlichen Welthorizont“ ist dadurch beibehalten, dass hier ein „differentielles System möglicher Erfahrungen“ in Anspruch genommen wird. Dabei stellt sich jedoch Tengelyi die Aufgabe, den husserlschen Ansatz so weiterzuführen, dass „das Dingreale mit dieser Dingstruktur richtig zu verbinden“ ist. Was bei Husserl fehlgeht, ist dabei keineswegs sein Ansatz beim „aktuellen Bewusstsein“, beim „Verhältnis des jeweiligen Ichsubjekts zu diesem System “43 oder bei „seinem ichlichen Können“44. Zu berichtigen ist vielmehr eine „Gleichsetzung [der Welt als] des Gesamthorizonts der Erfahrung mit einem bloßen Korrelat des Bewusstseins“: „Als Könnenshorizont

\footnotetext{
Vgl. dazu Römer Inga, „László Tengelyi - Die Welt und ihr Unendliches“, in Keiling T. (Hrsg.) Phänomenologie und Metaphysik der Welt (Arbeitstitel; in Vorbereitung).

40 Tengelyi L., Welt und Unendlichkeit, op. cit., S. 184.

41 Ibid., S. 299.

42 Ibid., S. 301.

43 Ibid., S. 538.

44 Ibid., S. 540.
} 
erweist sich die Gesamtwirklichkeit in Husserls Augen als ,eine Korrelatidee zur Idee einer vollkommenen Erfahrungsevidenz' [... “" 45 .

Das klassische Problem, dass nämlich die Welt letzten Endes ein bloßes Korrelat des Bewusstseins und sonst nichts bzw. nichts darüber hinaus sei, wird hier erneut zu einer Herausforderung. Wie kann man mit Husserl dazu gelangen, die Welt nicht in ein bloßes Korrelat des Bewusstseins zu verwandeln? Was steht dem im Wege? Nach Tengelyi muss auf seine Idee der Wirklichkeit des Dinges verzichtet werden, „der zufolge das Ding in der Welt an sich vollständig bestimmt ist“. Die „diakritische Aufgabe, die Idee eines unendlichen Systems möglicher Erfahrungen von der Seinstotalität des Dinges und der Gesamtwirklichkeit der Welt zu unterscheiden, mutet uns“, so Tengelyi, tatsächlich „einen Bruch“ mit dieser Idee zu. Aber anstatt sich von Husserl nun einfach abzukehren, findet Tengelyi bei ihm selbst einen Ausweg:

Deshalb bildet er [Husserl] auch den Begriff einer ,Erkenntniskontingenz' der Welt. Gemeint ist mit diesem Ausdruck keineswegs etwa ein Rückzug auf eine Erkenntnistheorie, die auf ontologische Einsichten aus methodischen Gründen verzichtet, sondern eine phänomenologische Besinnung auf die Erfahrungsgegebenheit der Welt, die jeden Versuch, Urtatsachen auf erste Ursachen zurückzuführen, von vornherein in den Bereich metaphysischer Abenteuer und spekulativer Überschwänglichkeiten verweist. ${ }^{46}$

Das scheint auch gegen Finks spekulative Denkweise gerichtet zu sein. Tengelyi kritisiert Finks These von der Notwendigkeit der Welt und mobilisiert stattdessen seine sich auf Husserl stützende These vom notwendigen Faktum der Welthabe. Er führt dabei die bereits erwähnte diakritische Methode ein, nach welcher die Unendlichkeit möglicherweise nicht erst durch die Subjektivität, für die es Erscheinen gibt, als Unendlichkeit des Erscheinenden in die Welt kommt. Denn diese Unendlichkeit der Welt wird durch die Offenheit der Weltwirklichkeit selbst ermöglicht, von der ja die diakritische Methode behauptet, sie gebe sich durch die Unverfügbarkeit kund, die eben mit der Erfahrung von Welt verbunden ist, und die das aktuelle System der Unendlichkeit möglicher Erfahrungen (also die Weltapperzeptionen in ihren Einstimmigkeiten) stört. Das heißt, sie bringt ihnen gegenüber ein Element der Alterität ins Spiel, das zwar in die Erfahrung aufgenommen, als ihre Kontingenz erlebt wird, aber in ihr nie absorbiert, aufgelöst

45 Idem.

46 Ibid., S. 324. Vgl. ibid.: „Der phänomenologische Charakter der Analyse ergibt sich hier [bei Husserl] daraus, dass die Erkenntniskontingenz der Welt mit der Beschreibung des Erfahrungsprozesses von vornherein in Verbindung gebracht wird." 
werden kann. Es ist die Offenheit der Welt, die bei Tengelyi diese Alterität, die als Kontingenz der Erfahrung erlebt wird, in sich schließt, und die somit noch von der Erfahrung aus phänomenologisch ausgewiesen werden kann, anstatt spekulativ über die Phänomenologie hinauszuweisen.

\section{Schluss}

Der auf die Faktizität der Welt-Vorgegebenheit gelegte Akzent bei Husserl, Landgrebe und Tengelyi, gegen die Tendenz, diese sowie die anderen Urtatsachen in die „letzte Faktizität“ eines notwendigen Bezugs auf das Absolute der Bewegung der Welt selbst einzubeziehen (wie es bei den Autoren der kosmologischen Wenden geschieht) und sie darin aufzulösen, macht aus dem Projekt einen Weg zurück zur Welt als Sache der Phänomenologie. Hier hat László Tengelyi mit seinem letzten Buch eine wichtige Schneise geschlagen. Wir können Aspekte dieses Weges aber auch bei Maurice Merleau-Ponty, Jan Patočka, Klaus Held und anderen Autoren finden, die wir hier nicht eingehend berücksichtigt haben. Sie knüpfen damit trotz kritischer Abstandnahme an die Weltproblematik bei Husserl an, von der wir ausgegangen sind, wobei sich ihre innovativen Relektüren einer Phänomenologie der Welt eben auch in Auseinandersetzung mit Ansätzen zu einer kosmologischen Wende artikulieren konnten, von denen hier vor allem derjenige Finks besprochen wurde.

Karel Novotný works in the Institute of Philosophy of the Czech Academy of Sciences and at the Faculty of Humanities of the Charles University in Prague. Co-director of the Central-European Institute of Philosophy. Erasmus Master Mundus "Europhilosophie" program local coordinator. Author of numerous studies, several editions and four book monographs, including La genèse d'une hérésie. Monde, corps et histoire dans la pensée de Jan Patočka (Paris: Vrin 2012) and Neue Konzepte der Phänomenalität. Essais zur Subjektivität und Leiblichkeit des Erscheinens (Würzburg: Königshausen und Neumann 2012).

E-mail: novotnykcz@yahoo.de 